\title{
III-V/Silicon-on-Insulator Photonic Integrated Circuit for Fiber-to- the-Home Central Office Transceivers in a Point-to-Point Network Configuration
}

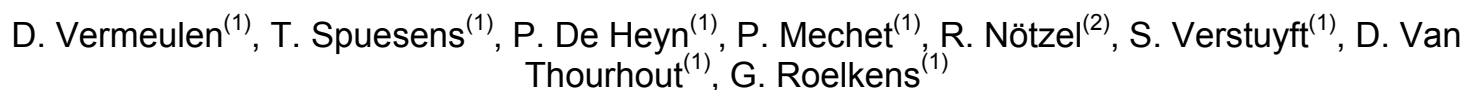 \\ (1) Photonics Research Group, Ghent University/IMEC, Sint-Pietersnieuwstraat 41, B-9000 Ghent, \\ Belgium, diedrik.vermeulen@intec.ugent.be \\ (2) Photonics and Semiconductor Nanophysics, Eindhoven University of Technology, The Netherlands
}

\begin{abstract}
We describe the realization of III-V/silicon-on-insulator photonic integrated circuits for Fiberto-the-Home transceivers in the central office. Above 0.4A/W responsivity is obtained in the $1310 \mathrm{~nm}$ band (with polarization dependent loss below 0.5dB). Crosstalk from the $1490 \mathrm{~nm} / 1550 \mathrm{~nm}$ wavelength channel is below $-20 d B$.
\end{abstract}

\section{Introduction}

In the Fiber-to-the-Home (FTTH) market, two technologies are competing: passive optical networks (PON) and Ethernet-based point-to point networks. The European market is mostly choosing Ethernet-based point-to-point. The Japanese and - to a lesser extent - the US markets are working with PON. Ethernet pointto-point has considerable advantages over PON: Ethernet is an open standard, upgrading is easier and the network is future-proof. However, PON has a strong advantage as well: since PON networks are shared between endusers, the space and power consumption required for the central office is much smaller. In addition, the cost of the PON central office equipment is less than for point-to-point access switches. Reducing the size, power consumption and cost of Ethernet-based central office equipment, which consists of an individual transceiver per user, would therefore take away important drawbacks of point-to-point networks.

Photonic integrated circuits allow integrating multiple optical components on a single chip, thereby realizing the required reduction in size, cost and power consumption for the central office equipment. Contrary to traditional platforms for photonic integration such as III-V monolithic integration, which requires expensive regrowth techniques, or silica-on-silicon, which is not compatible with a compact form-factor, the silicon platform seems the only one which is truly compatible with large scale integration. This is due to the high refractive index contrast that is available in so called silicon photonic wires, allowing for wavelength scale routing and handling of light on the SOI chip. Wafer-level processing using CMOS fabrication tools and wafer level testing will enable low cost levels.
Using high index contrast photonic integrated circuits for communication applications however also brings along considerable challenges, especially regarding the efficient fiber-to-chip coupling and polarization independent operation of the photonic integrated circuit. Especially since the photonic integrated circuit needs to be able to handle widely spaced wavelength bands. Efficient optical coupling $(-1.6 \mathrm{~dB})$ from a single mode fiber to a silicon photonic wire was demonstrated $^{1}$ using a diffractive grating structure for a single polarization in the optical fiber. The $1 \mathrm{~dB}$ optical bandwidth is limited to $50 \mathrm{~nm}$ however. In order to address the polarization sensitivity of the grating coupler, a polarization diversity approach was presented ${ }^{2}$ using two-dimensional grating couplers to couple both orthogonal polarizations to the silicon chip. While this allows reducing the polarization dependent loss, it also only works over a limited wavelength range, insufficient for Fiber-to-the-Home transceiver applications, where a $100 \mathrm{~nm}$ wavelength band needs to be covered in the $1310 \mathrm{~nm}$ wavelength range). Moreover, the two-dimensional grating approach introduces a decrease in fiber-to-chip coupling efficiency.

Since the FTTH transceivers require photodetection and light emission on the photonic integrated circuit, other materials need to be integrated in order to perform these functions. Ge-based photodetectors are a good option given their direct compatibility with the CMOS compatible processing of the silicon photonic integrated circuit. In this paper however we focus on a novel photodetector configuration based on a heterogeneously integrated III-V semiconductor photodetector. This approach has the advantage that it also allows the 
integration of III-V light sources and III-V modulators on the same photonic integrated circuit.

\section{Transceiver configuration}

The layout of the considered device is schematically depicted in figure 1 . The III-V semiconductor layer stack consists of an InP/InGaAsP p-i-n structure with an absorption layer with a band gap wavelength of $1.37 \mu \mathrm{m}$. This bandgap wavelength allows the $1310 \mathrm{~nm}$ wavelength band to be efficiently absorbed, while the absorption layer is transparent for the $1490 \mathrm{~nm}-1550 \mathrm{~nm}$ wavelength channels. In order for the detector to be completely transparent for these wavelength channels, the III-V layer stack thicknesses have been optimized for transparency at the central wavelength of $1520 \mathrm{~nm}$. The III-V heterostructure is transferred on top of a silicon grating coupler structure using DVS-BCB adhesive wafer bonding ${ }^{3}$. After layer transfer, the III-V photodetector is processed, lithographically aligned to the underlying siliconon-insulator waveguide circuit. The main idea underlying this configuration is that it allows efficient detection with low polarization dependent loss over the full 1260nm-1360nm wavelength band, since this light is not coupled to the silicon waveguide layer. On the other hand, the $1490 \mathrm{~nm} / 1550 \mathrm{~nm}$ wavelength channels (which are generated on chip and therefore have controlled polarization and better controlled emission wavelength) can be efficiently coupled from the silicon waveguide layer to the optical fiber using a one-dimensional diffractive structure (operating for a single polarization) ${ }^{1}$.

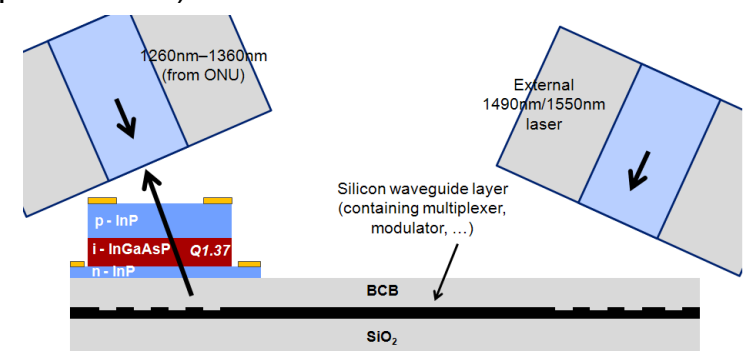

Fig. 1: Schematic cross-section of the III-V/SOI transceiver

A microscope image of a prototype device is depicted in figure 2. The III-V photodetector was processed on top of a silicon-on-insulator waveguide circuit consisting of a planar concave grating capable of multiplexing a 1490nm-band data signal and a $1550 \mathrm{~nm}$-band data signal in a single output waveguide. The devices were fabricated using standard CMOS fabrication tools on an 8inch SOI wafer consisting of a
$220 \mathrm{~nm}$ silicon waveguide layer and $2 \mu \mathrm{m}$ buried oxide layer thickness (through the multi-project wafer shuttle run service ePIXfab).

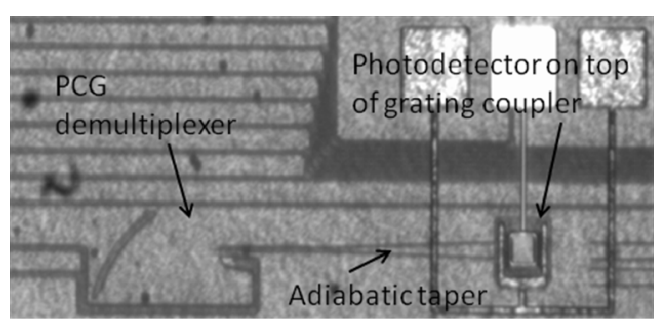

Fig. 2: Microscope image of the fabricated IIIVISOI transceiver circuit

This serves as a proof-of-principle that the silicon waveguide circuit can contain functional elements that handle $1490 \mathrm{~nm}$ and $1550 \mathrm{~nm}$ wavelength bands. In the future we will extend the functionality on the silicon platform by adding integrated power splitters, arrays of $1490 \mathrm{~nm}$ and $1550 \mathrm{~nm}$ modulators and wavelength multiplexers to realize integrated transceiver arrays (e.g. 8 channels) for central office equipment with a single $\mathrm{CW}$ external light source (VCSEL or DFB laser) per wavelength band, as shown in figure 3 .

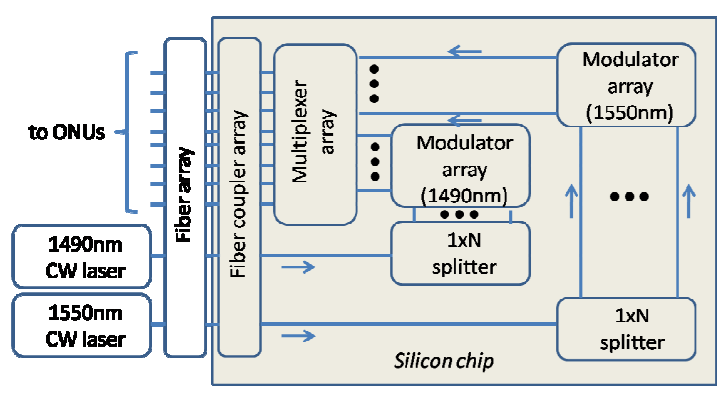

Fig. 3: Envisioned device configuration for integrated transceiver arrays for FTTH central office equipment

\section{Transceiver characterization}

The fabricated devices were characterized in the $1310 \mathrm{~nm}$ and $1490-1550 \mathrm{~nm}$ wavelength band. For this proof-of-principle device, a standard silicon diffractive grating structure was used with a known fiber-to-chip coupling efficiency of $-6 \mathrm{~dB}$. The implementation of advanced fiber-to-chip couplers ${ }^{1}$ with a fiber-to-chip coupling efficiency of $-1.6 \mathrm{~dB}$ is expected not to affect the operation principle of the proposed device. The optical fiber is tilted 10 degrees off vertical in order to avoid second order Bragg reflection from the silicon grating. Figure $4 a$ shows the responsivity of the photodetector for the $1310 \mathrm{~nm}$ wavelength band. It varies around $0.4 \mathrm{~A} / \mathrm{W}$. The polarization dependent loss is plotted in figure $4 \mathrm{~b}$, showing excellent behaviour ( $<0.5 \mathrm{~dB} P D L)$ over the full wavelength range. 


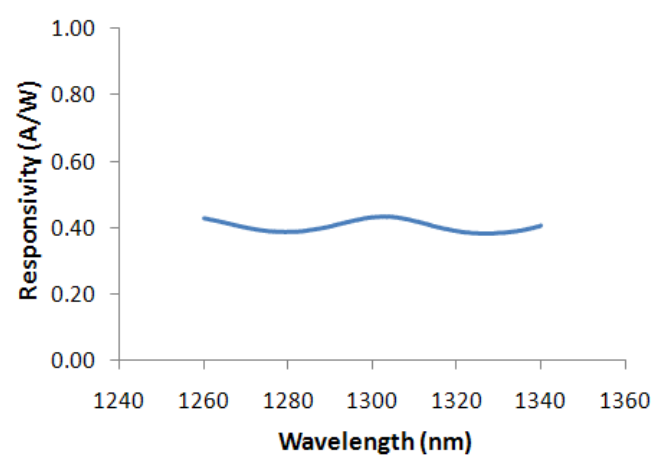

(a)

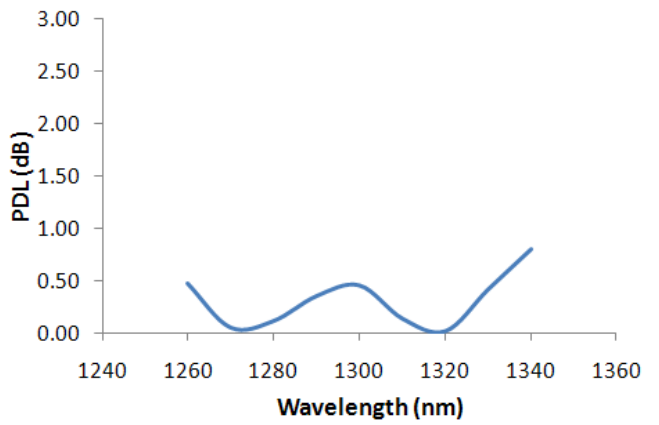

(b)

Fig. 4: Photodetector responsivity (a) in the $1310 \mathrm{~nm}$ wavelength band and the associated polarization dependent loss (PDL) (b)

This would have been very hard to obtain when the $1310 \mathrm{~nm}$ channel had to be coupled to the silicon waveguide circuit and demultiplexed from the $1490 \mathrm{~nm} / 1550 \mathrm{~nm}$ signal prior to photodetection. Assuming an external laser source for the $1490 \mathrm{~nm} / 1550 \mathrm{~nm}$ upstream channel (from the viewpoint of the central office equipment), the fiber-to-fiber transmission of the fabricated photonic IC (using two identical grating couplers of which one is covered by the heterogeneously integrated photodetector) was measured. $-12 \mathrm{~dB}$ peak transmission, consistent with the $-6 \mathrm{~dB}$ fiber-to-chip coupling efficiency of a single grating, was obtained. This shows that the integration of the photodetector on top of the fiber coupler doesn't aversely affect its performance in the $1490 \mathrm{~nm} / 1550 \mathrm{~nm}$ wavelength range.

In order to assess if the integrated photodetector is truly transparent for the $1490 \mathrm{~nm} / 1550 \mathrm{~nm}$ wavelength channel and doesn't introduce a significant electrical crosstalk in the photodetector, the device responsivity was also characterized in the 1490nm/1550nm wavelength band. This crosstalk, here defined as the ratio between the responsivity at a wavelength in the $1490 \mathrm{~nm} / 1550 \mathrm{~nm}$ wavelength range to a typical responsivity of $0.4 \mathrm{~A} / \mathrm{W}$ in the $1310 \mathrm{~nm}$ wavelength range is shown in figure 5 , and is below $-30 \mathrm{~dB}$. Therefore, even a significant difference in optical power between the point-to-point downstream 1310nm wavelength channel and the upstream $1490 \mathrm{~nm} / 1550 \mathrm{~nm}$ wavelength channel of $10 \mathrm{~dB}$ will still lead to better than $-20 \mathrm{~dB}$ crosstalk in the photodetector, thereby showing the feasibility of this approach. The planar concave grating, used to multiplex the $1490 \mathrm{~nm}$ and $1550 \mathrm{~nm}$ wavelength channels works as expected after photodetector integration (although with a higher insertion loss due to the cladding of the device with DVS-BCB, which reduced the power reflection at the silicon grating facets, but this can be accounted for in the design).

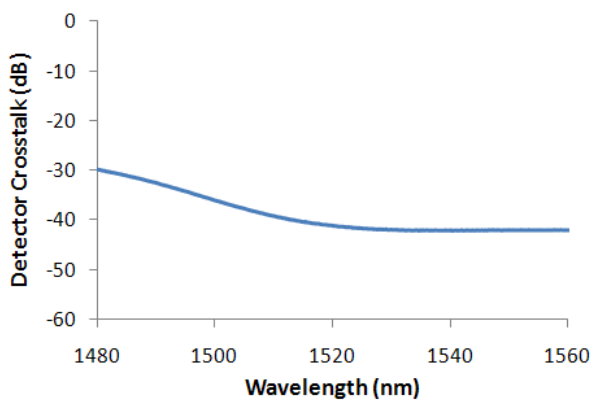

Fig. 5: Photodetector cross-talk due to the transmission of the $1490 \mathrm{~nm} / 1550 \mathrm{~nm}$ data signal

At the conference, more data will be presented regarding the dynamic performance of the photodetector using modulated data signals.

\section{Conclusions}

This paper shows for the first time that a heterogeneous III-V/silicon photonic integrated circuit can be used to realize integrated transceiver arrays for Fiber-to-the-Home central office equipment in point-to-point networks. The concept is limited to operation in the central office, but there, the need for integration - in order to reduce the floor space and power consumption in the central office - is the most obvious.

\section{Acknowledgements}

This work was carried out in the framework of the Dutch Smartmix-Memphis project. D. Vermeulen and T. Spuesens thank the Institute for the Promotion of Innovation by Science and Technology in Flanders (IWT) for a grant. G. Roelkens acknowledges the Fund for Scientific Research Vlaanderen (FWO) for a postdoctoral grant.

\section{References}

1 D. Vermeulen et al., Proc. Group IV Photonics p. FPD1 (2009)

2 R. Halir et al,. Proc. OFC'10, p. OWJ1 (2010)

3 G. Roelkens et al., Jorn. Electrochem. Soc. p. G1015-G1019 (2006) 